\title{
On the performance of Usain Bolt in the 100 metre sprint
}

\author{
JJ Hernández Gómez, V Marquina and RW Gómez \\ Facultad de Ciencias, Universidad Nacional Autónoma de México. \\ Circuito Exterior C.U. México D.F., 04510, México \\ E-mail: jorge_hdz@ciencias.unam.mx, marquina@unam.mx, rgomez@unam.mx
}

\begin{abstract}
Many university texts on Mechanics deal with the problem of the effect of the air drag force, using as example the slowing down of a parachute. Hardly no one discuss what happens when the drag force is proportional to both $u$ and $u^{2}$. In this paper we deal with a real problem to illustrate the effect of both terms in the speed of a runner: a theoretical model of the performance of the $100 \mathrm{~m}$ world record sprint of Usain Bolt during the 2009 World Championships at Berlin is developed, assuming a drag force proportional to $u$ and to $u^{2}$. The resulting equation of motion is solved and fitted to the experimental data obtained from the International Amateur of Athletics Federations that recorded Bolt's position with a LAVEG (laser velocity guard) device. It is worth to note that our model works only for short sprints.
\end{abstract}

PACS numbers: 01.80.tb, 01.55.tb, 45.20.D, 07.10-h, 06.30.Bp

Keywords: Usain Bolt, mechanical model, hydrodynamic drag, sport physics.

Submitted to: Eur. J. Phys. 


\section{Introduction}

In June 21, 1960, at Zürich, Switzerland, the German Armin Harry astounded the sports world achieving what was considered the physiological and psychological barrier for the $100 \mathrm{~m}$ dash: 10 s flat. It was until June 20, 1968, at Sacramento, California, that Jim Hines ran $100 \mathrm{~m}$ in 9.9 $\mathrm{s}$, breaking this barrier. In following years many sprinters had run this distance faster than 10 s, but 31 years were needed to lower Harry's record by $0.14 \mathrm{~s}$ (Carl Lewis, August 25, 1991, at Tokyo, Japan). The current world record of $9.58 \mathrm{~s}$ was established by Usain Bolt (who also held the $200 \mathrm{~m}$ world record of $19.19 \mathrm{~s}$ up to 2012) in the 12th IAAF World Championships in Athletics at Berlin, Germany (2009).

The performance of Usain Bolt in the 100 meter sprints is of physical interest since he can achieve, until now, accelerations and speeds that no other runner can. Through time, several mathematical models to fit the position, the velocity, or both, of a sprinter have been proposed [1, 2, 3, 4, 5, 6]. Recently, Helene et al [6] fitted Bolt's performance during both the summer Olympics in 2008 at Beijing and the world championships in 2009 at Berlin, using a simple exponential model for the time dependence of the speed of the runner.

\section{Theoretical model}

The important forces acting during the race are the horizontal force that Bolt exerts and a drag force that depends upon the horizontal velocity (speed). Other factors, such as humidity, altitude above sea level ( $36 \mathrm{~m}$ ), oxygen intake and that he turns his head to watch other runners, affecting the mechanics of his motion, are not taken into account. Based on the fact that Bolt's $200 \mathrm{~m}$ time is almost twice the one for $100 \mathrm{~m}$, our main assumption is that in the $100 \mathrm{~m}$ dash, he is able to develop a constant horizontal force $F_{0}$ during the whole sprint. The drag force, $D(u)$, is a function of Bolt's horizontal speed respect to ground $u(t)$, with or without wind. This force causes a reduction of his acceleration so his speed tends to a constant value (terminal speed). Thus, the equation of motion is

$$
m \dot{u}=F_{0}-D(u) .
$$

This equation can be readily casted as a quadrature,

$$
t-t_{0}=m \int_{u_{0}}^{u} \frac{d u^{\prime}}{F_{0}-D\left(u^{\prime}\right)} .
$$


The integral above does not have an analytical solution for a general drag function; however the drag force can be expanded in Taylor series,

$$
D(u) \simeq D(0)+\left.\frac{d D(u)}{d u}\right|_{0} u+\left.\frac{1}{2} \frac{d^{2} D(u)}{d u^{2}}\right|_{0} u^{2}+O\left(u^{3}\right) .
$$

The constant term of the expansion is zero, because the runner experiments no drag when at rest. The second and third terms must be retained. While the term proportional to the speed represents the basic effects of resistance, the term proportional to the square of the speed takes into account hydrodynamic drag, obviously present due to the highly non-uniform geometry of the runner. In general, for relatively small speeds, it suffices to take only the first three terms of the expansion.

Renaming the $u$ and $u^{2}$ coefficients as $\gamma$ and $\sigma$ respectively, the equation of motion (1) takes the form

$$
m \dot{u}=F_{0}-\gamma u-\sigma u^{2},
$$

whose solution follows straightforward from Equation (2),

$$
u(t)=\frac{A B\left(1-e^{-k t}\right)}{A+B e^{-k t}},
$$

where the coefficients are related by $\sigma=k m /(A+B), F_{0}=k m A B /(2 A+2 B)$ and $\gamma=$ $k m(A-B) /(A+B)$.

The position can be obtained by integrating Equation (5),

$$
x(t)=\frac{A}{k} \ln \left(\frac{A+B e^{-k t}}{A+B}\right)+\frac{B}{k} \ln \left(\frac{A e^{k t}+B}{A+B}\right),
$$

while the acceleration can also be calculated by deriving Equation (5),

$$
a(t)=A B k(A+B) \frac{e^{-k t}}{\left(A+B e^{-k t}\right)^{2}} .
$$

\section{Experimental data fitting}

The experimental data we used were from the 12th IAAF World Championships in Athletics (WCA), which were obtained from [7], and consist of Bolt's position and speed every 1/10 s. To corroborate the accuracy of the data obtained from [7], we reproduced with them the velocity vs position plot given in [8], which was obtained by the IAAF, by means of a LAVEG (laser velocity guard) device. The parameters $A, B$ and $k$ were fitted by a least-squares analysis, with the Origin 8.1 (C) software, in both position and speed data sets, considering a reaction time of 
$0.142 \mathrm{~s}$ [6]. In figure 1(a) and figure 1(b) we show such fittings, together with the experimental data.

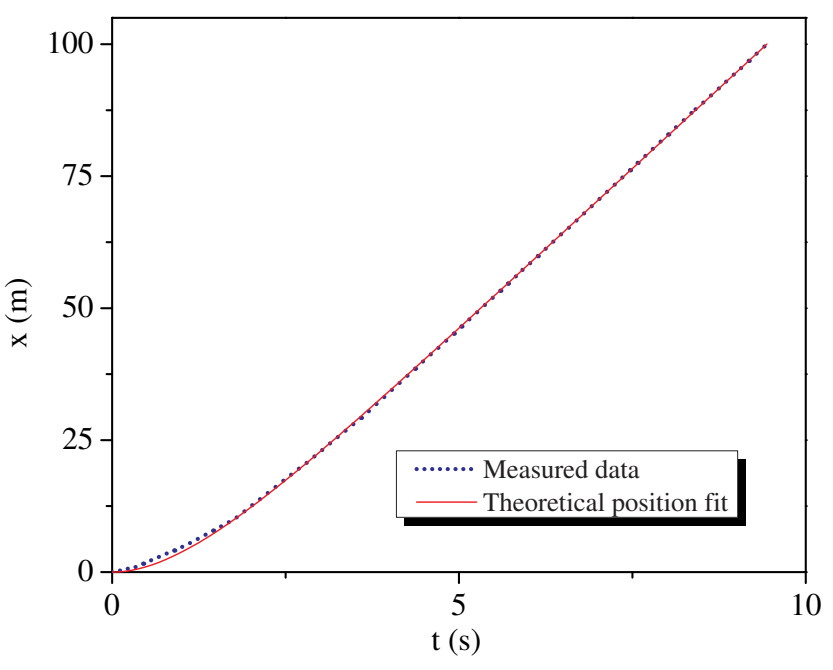

(a)

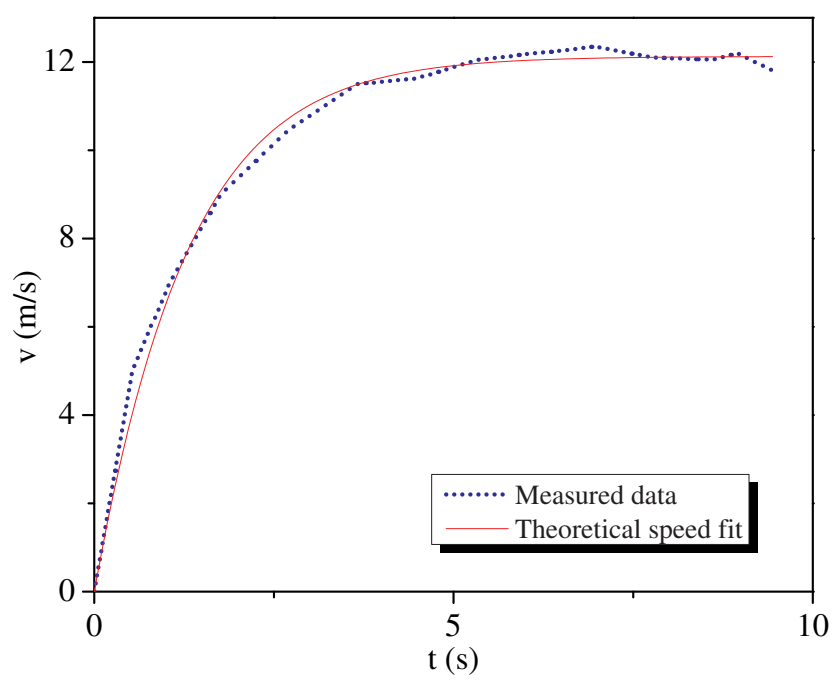

(b)

Figure 1: Position (a) and speed (b) of Bolt in the $100 \mathrm{~m}$ sprint at the 12th IAAF WCA. The dotted (blue) line corresponds to the experimental data while the solid (red) one corresponds to the theoretical fitting.

The parameter values for both fittings are shown in table 1. We do not report errors, because the standard error of the fitting on each parameter lies between the second and the third significant digit, which is finer than the measurement error in the data.

Table 1: Fitted values of the parameters $A, B$ and $k$.

\begin{tabular}{ccc}
\hline Parameter & Position fitting & Velocity fitting \\
\hline$A(\mathrm{~m} / \mathrm{s})$ & 110.0 & 110.0 \\
$B(\mathrm{~m} / \mathrm{s})$ & 12.2 & 12.1 \\
$k(1 / \mathrm{s})$ & 0.9 & 0.8 \\
\hline
\end{tabular}

The accuracy of the position and velocity fittings is $R_{p}^{2}=0.999$ and $R_{v}^{2}=0.993$ respectively, so we decided to use from now on the results of the parameters $A, B$ and $k$ from the position fitting. The computed values of the magnitude of the constant force $F_{0}$, and the drag coefficients, $\gamma$ and $\sigma$, are shown in table 2, taking Bolt's mass as $86 \mathrm{~kg}$ [9].

We also show in figure 2 the plot of the magnitude of the acceleration we obtained; no fitting was made because there are no experimental data available. 
Table 2: Values of the physical parameters $F_{0}, \gamma$ and $\sigma$.

\begin{tabular}{cc}
\hline Constant & Value \\
\hline$F_{0}(\mathrm{~N})$ & 815.8 \\
$\gamma(\mathrm{kg} / \mathrm{s})$ & 59.7 \\
$\sigma(\mathrm{kg} / \mathrm{m})$ & 0.6 \\
\hline
\end{tabular}

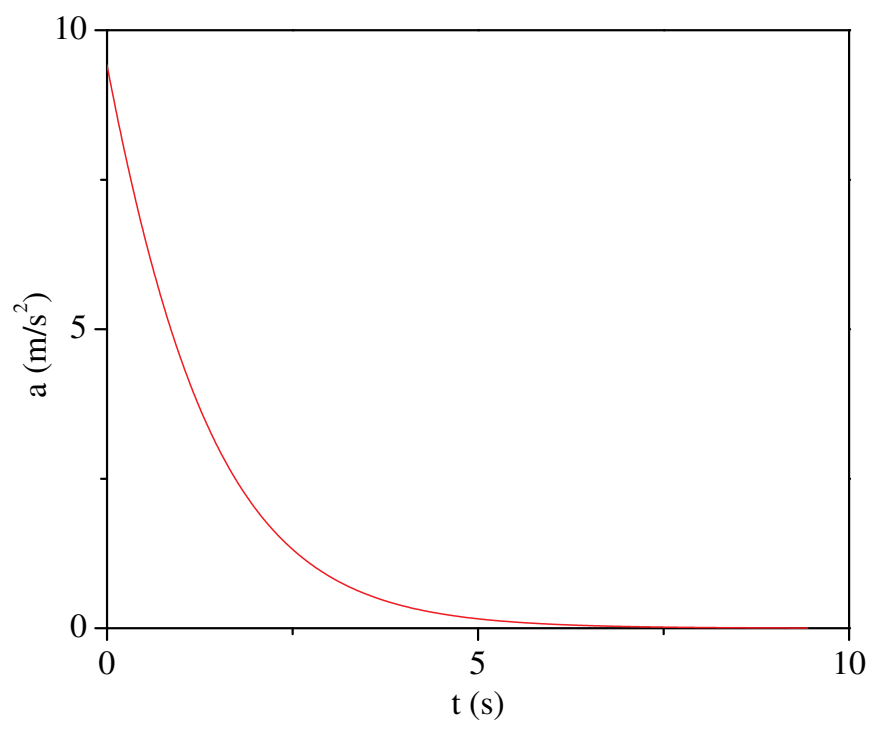

Figure 2: Theoretical acceleration of Bolt in the $100 \mathrm{~m}$ sprint at the 12th IAAF WCA.

\section{Results}

As any mechanical system subject to drag, the runner experiments a terminal velocity $u_{T}$ which is formally obtained when $\dot{u}=0$ in the equation of motion (1); that is, by solving the equation

$$
F_{0}=D\left(u_{T}\right)
$$

for $u_{T}$. Nevertheless, the solution of the equation for the terminal velocity can also be found when $t \rightarrow \infty$ in Equation (5), and it turns out to be $u_{T}=B$. Therefore, under this model, the runner acquires a terminal speed of $u_{T}=12.2 \mathrm{~m} / \mathrm{s}$, which is physically feasible (see figure [1(b)]. According to the data obtained from [7], the average speed in the second half of the sprint, which is surprisingly equal to $99 \%$ of the maximum speed recorded [7], is 12.15 $\mathrm{m} / \mathrm{s}$. Moreover, the initial acceleration of Bolt is $a(0)=9.5 \mathrm{~m} / \mathrm{s}$, which is of the order of the acceleration of gravity, $g$; this value of the initial acceleration is fully reasonable, considering that the acceleration a man must exert in order to be able to jump half of his own height, should 
be just slightly greater than $g$. Furthermore, the value of the constant force in table $2, F_{0}=815.8$ $\mathrm{N}$, is totally consistent with the fact that one expects that the maximum constant (horizontal) force he could exert should be of the order of his weight, i.e. $w=842.8 \mathrm{~N}$.

Now, $\sigma=0.5 \rho C_{d} A$ represents the hydrodynamic drag, where $\rho$ is the density of air, $C_{d}$ the drag coefficient of the runner and $A$ his cross section area. The density of air at the time of the spring can be approximated as follows. Berlin has a mean altitude of $34 \mathrm{~m}$ over the sea level, and an average mean temperature for the month of August [10] of $18.8^{\circ} \mathrm{C}$. Bearing in mind that the race took place at night, we consider an average temperature between the average mean temperature and the mean daily minimum temperature for August at Berlin, which is $14.3^{\circ} \mathrm{C}$. Thus, the density of air is $\rho=1.215 \mathrm{Kg} / \mathrm{m}^{3}$ and the drag coefficient of Bolt is $C_{d}=2 \sigma / \rho A=1.2$, where the cross section area of Bold was estimated as $A=0.8 \mathrm{~m}^{2}$. This value of $C_{d}$ lays in the typical range for human beings reported in the literature (between 1.0 and 1.3) [11, 12, 13].

The instantaneous power that Bolt develops, considering the drag effect is simply

$$
P(t)=F u=m \dot{u} u=m A B k(A+B) \frac{\left(1-e^{-k t}\right) e^{-k t}}{\left(A+B e^{-k t}\right)^{3}} .
$$

In figure 3 we plot the power of the sprint for Bolt and the drag. It is remarkable that the maximum power of $P_{\max }=2619.5 \mathrm{~W}(3.5 \mathrm{HP})$ is reached at a time of $t_{P \max }=0.89 \mathrm{~s}$, when the speed $u\left(t_{P_{\max }}\right)=6.24 \mathrm{~m} / \mathrm{s}$ was only about half of the maximum speed. The fact that the maximum instantaneous power arises in such a short time indicates the prompt influence of the drag terms in the dynamics of the runner.

The effective work (considering the effect of the drag force) is then

$$
W_{\mathrm{Eff}}=\int_{0}^{\tau} P(t) d t=\int_{0}^{\tau} \frac{1}{2} m d u^{2}=\frac{1}{2} m u^{2}(\tau)
$$

where $\tau$ is the running time (the official time of the sprint minus the reaction time of the runner). The effective work is the area under the curve of figure 3, and it is $W_{\mathrm{Eff}}=6.36 \mathrm{~kJ}$. On the other hand, as Bolt is assumed to develop an essentially constant force, his mechanical work is simply $W_{\mathrm{B}}=F_{0} d=81.58 \mathrm{~kJ}$, where $d$ is the length of the sprint $(100 \mathrm{~m})$. This means that from the total energy that Bolt develops, only $7.79 \%$ is used to achieve the motion, while $92.21 \%$ is absorbed by the drag; that is, $75.22 \mathrm{~kJ}$ are dissipated by the drag, which is an incredible amount of lost energy.

$\ddagger$ The sprint took place on August 16, 2009.

$\S$ To calculate such a cross section area, we used a similar procedure to the one used in [9], where instead of a circle we estimated the area of the head with an ellipse. We averaged several scaled measures from Bolt pictures taken from [14] 


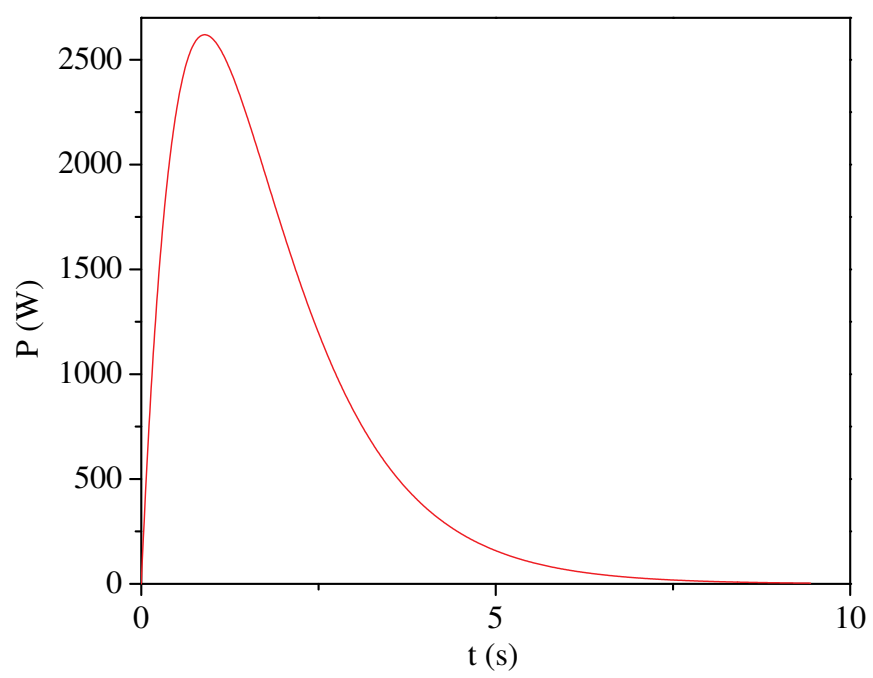

Figure 3: Theoretical power of Bolt in the $100 \mathrm{~m}$ sprint at the 12th IAAF WCA.

\section{Discussion}

As mentioned in section 2, a central assumption in our model is that a $100 \mathrm{~m}$ sprinter (not only Bolt) is able to develop a constant force during the race (except in the initial few tenths of a second where he pushes himself against the starting block). In order to delimit how good is this assumption, we use the experimental values of $u$, the calculated acceleration, and the fitted values of the constants $\gamma$ and $\sigma$, to compute $F_{0}$. The result is shown in figure 4, It is interesting to note that the average value of the force obtained from this figure is $818.3 \mathrm{~N}$, which is very close to the value obtained from the fitting of the data, $815.7 \mathrm{~N}$. The high value of the force in the first tenths of the race is due do the acceleration he obtains when he pushes himself from the starting block.

At a first glance, observing the values of the drag coefficients in table 2, one is impelled to argue that, because $\sigma \ll \gamma$, the hydrodynamic drag could have been neglected. However, one can calculate the drag terms in the equation of motion at the terminal speed $u_{T}$, attaining $\gamma u_{T}=725.59 \mathrm{~N}$ and $\sigma u_{T}{ }^{2}=90.18 \mathrm{~N}$. Thus, from the total drag $\gamma u_{T}+\sigma u_{T}^{2}, 11.05 \%$ corresponds to turbulent drag, which turns to be an important contribution.

If we would like to make predictions considering different wind corrections, this can be done as follows. Once a runner acquires the wind speed (which is almost instantly), the second term in the right side $(\gamma u)$ of equation (1) behaves as if the sprinter would be running in still air, because $\gamma$ is proportional to the air viscosity, which is independent of air pressure. However that is not the case for the third term in (1), $\left(\sigma u^{2}\right)$, which arises from the collisions per unit time of the air molecules against the sprinter and it is proportional to the speed of the runner respect to ground. In a simple model, the hydrodynamical drag force is $D_{H}=\sigma(\rho)\left(v+v_{w}\right)^{2}$, where $v$ is 


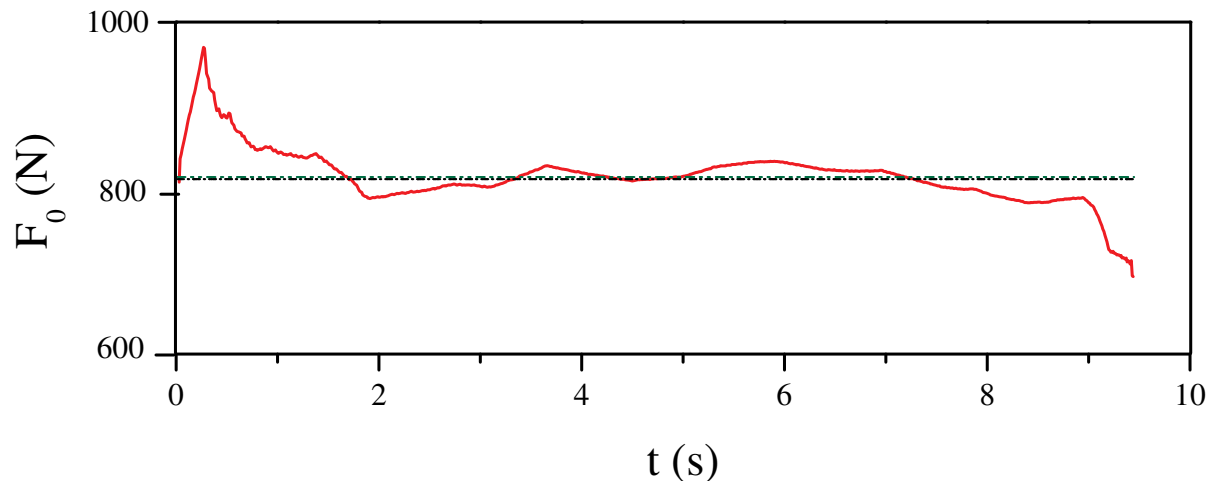

Figure 4: Force exerted by the runner during the race. The red line is calculated with the experimental data, the dash-dot-dot (green) line is the average force of $818.3 \mathrm{~N}$, while the short-dash-dot (black) line is the value of the force $F_{0}$ obtained from the adjustment $(815.7 \mathrm{~N})$.

the speed achieved by the runner without wind and $v_{w}$ is the speed of the wind. The value of $\sigma$ depends on the number of molecules that impact on the runner per unit time and should be different in still air conditions. Then, the equation of motion (1) can be rewritten as

$$
m \dot{u}=m \dot{v}=F_{0}-\gamma v-\sigma\left(v+v_{V}\right)^{2},
$$

and without wind as

$$
m \dot{v}=F_{0}-\gamma v-\sigma^{\prime} v^{2} .
$$

Subtracting (11) and (12), we obtain

$$
\sigma\left(v^{2}+2 v v_{w}+v_{w}^{2}\right)=\sigma^{\prime} v^{2},
$$

so then

$$
\sigma^{\prime}=\sigma\left(1+\frac{2 v_{w}}{v}+\frac{v_{w}^{2}}{v^{2}}\right) \sim \sigma\left(1+\frac{2 v_{w}}{v}\right),
$$

where the third term in the second expression has been neglected $\left(v_{w}<<v\right)$. In order to estimate the value of $\sigma^{\prime}$, we consider $v$ as the terminal speed of Bolt, $u_{T}$. With these conditions, $\sigma^{\prime}=0.69$ with still air $\left(v_{w}=0 \mathrm{~m} / \mathrm{s}\right)$ and $\sigma^{\prime}=0.49$ with a tailwind of $v_{w}=2 \mathrm{~m} / \mathrm{s}$. It should be clear that the present calculation is only a crude way to estimate the differences of running time with and without wind. The results, which are close to the values reported in literature[15], are summarized in table 3 ,

Although this is a simple way to calculate a correction due to wind, it turns to be a good proposal for it. A more realistic assumption would be to modify equation (14) to be

$$
\sigma^{\prime}=\sigma\left(1+\frac{\alpha v_{w}}{u_{T}}\right)
$$


Table 3: Predictions of the running time for Bolt without tailwind, and with a tailwind of $2 \mathrm{~m} / \mathrm{s}$.

\begin{tabular}{cc}
\hline $\boldsymbol{v}_{\boldsymbol{w}}(\mathrm{m} / \mathrm{s})$ & Estimated running time $(\mathrm{s})$ \\
\hline 0 & 9.68 \\
0.9 & 9.58 \\
2 & 9.46 \\
\hline
\end{tabular}

with the parameter $\alpha$ lying between 1 and 2 .

The results we obtained, altogether with the facts pointed out in this discussion, shows the appropriateness and quality of the model developed in this paper. We look forward for the next IAAF WCA, which will be held in Moscow, Russia, from August 10 to August 18, 2013, to test our model with the experimental data obtained from such sprints, as well as to wait expectantly if the fastest man on earth is able to beat his own world record once again.

\section{Acknowledgments}

This work was partially supported by PAPIIT-DGAPA-UNAM Project IN115612.

\section{References}

[1] J Keller. . A theory of competitive running. Phys. Tod. 9, 26, $42-47$ (1973). Reprinted in [16].

[2] I Alexandrov and P Lucht. Physics of sprinting. Am. J. Phys. 49, 254-257 (1981). Reprinted in [16].

[3] R Tibshirani. Who is the fastest man in the world?. Am. Stat., 51, 106-111 (1997).

[4] G Wagner. The 100-meter dash: Theory and experiment. Phys. Teach. 36, 144-146 (1998).

[5] A Heck and T Ellermeijer Giving students the run of sprinting models. Am. J. Phys. 77, 1028 (2009).

[6] O Helene and M Yamashita. The force, power and enery of the 100 meter sprint. Am. J. Phys. 78, 307 (2010).

[7] http://www.youtube.com/watch?v=SyY7RgNLCUk

[8] International Amateur Athletics Federation. Berlin Biomechanics Project. http://berlin.iaaf.org/news/ kind=101/newsid=53084.html

[9] JD Charles and A Bejan. The evolution of speed, size and shape in modern athletics. J. Exp. Biol. 212, 2419-2425 (2009).

[10] World Meteoroloical Organization. World Weather Information Service - Berlin. http://worldweather.wmo.int/016/c00059.htm

[11] J Shanebrook and R Jaszczak Aerodynamic drag analysis of runners. Med. Sci. Sports 8 1, 43-45 (1976).

[12] V Zatsiorsky Kinetics of human motion. (2002).

[13] L Brownlie. Aerodynamic and thermal characteristics of running apparel. Simon Fraser University (1982).

[14] Usain Bolt official website. http://usainbolt.com/

[15] JR Mureika. The Legality of wind and altitude assisted performance in the sprints New Studies in Athletics, 15, 53-60 (2008). 
[16] A. Armenti Jr. The physics of sports New York, American Institute of Physics (1992). 